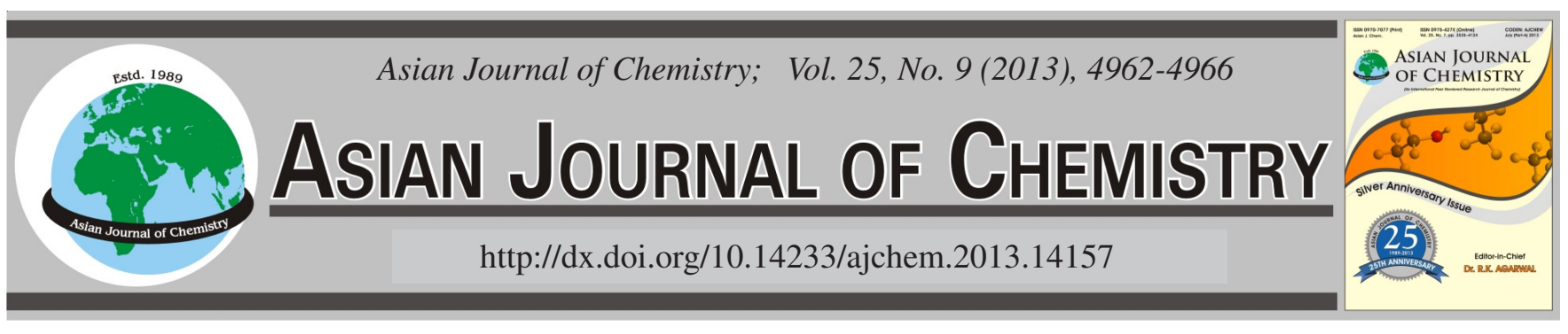

\title{
Molecular Dynamic Simulation Study on Thermal Decomposition Mechanism of Cellulose
}

\author{
Jinbao Hunang ${ }^{1, *}$, Hong Tong ${ }^{1}$, Guisheng Zeng ${ }^{2}, \mathrm{Yu}_{\mathrm{XIE}}{ }^{2,3}$ and Weimin $\mathrm{Li}^{1}$
}

${ }^{1}$ School of Science, Guizhou University for Nationalities, Guiyang 550025, P.R. China

${ }^{2}$ School of Environment and Chemical Engineering, Nanchang Hangkong University, Nanchang 330063, P.R. China

${ }^{3}$ State Key Laboratory of Pulp and Paper Engineering, South China University of Technology, Guangzhou 510640, P.R. China

*Corresponding author: Tel./Fax: +86 851 3610156; E-mail: huangjinbao76@126.com

(Received: 6 June 2012;

Accepted: 13 March 2013)

AJC-13113

\begin{abstract}
Cellulose is the most elementary component in biomass, of which thermal decomposition behaviour embodies the whole thermal decomposition law of biomass to a large extent. In order to ascertain the cellulose thermal decomposition mechanism and the formation mechanism of main products from the microscopic perspective, the thermal decomposition processes of cellulose single molecule with 10 monomers were investigated by molecular dynamic simulation method. The simulation results show that hydroxyl bonds begin to break when temperature reaches $450 \mathrm{~K}$. While as the temperature increases to $c a .600 \mathrm{~K}$, cellulose monomers get to be formed by glucoside bonds rupture; and pyranoid rings becomes open and all kinds of molecular fragments are formed at the same time. Based on the previous experimental results, the possible formation pathways of major products through reactions of all kinds of molecular fragments were analyzed.
\end{abstract}

Key Words: Molecular dynamic simulation, Cellulose, Thermal decomposition.

\section{INTRODUCTION}

With increasing concerns about energy supply and pollution problems caused by burning fossil fuels, the utilization of biomass resources which are renewable resources has attracted more and more concerns. The sulphur, nitrogen, ash content in biomass is low and biomass energy is one renewable and clean energy, which can reduce the greenhouse effect, so the biomass energy has become the focus of research globally ${ }^{1-5}$. The thermal decomposition behaviour of cellulose as the most basic component in biomass reflects the whole thermal decomposition law of biomass to a large extent, so the study on cellulose pyrolysis mechanism will be significant to understand biomass pyrolysis mechanism.

Cellulose is the most abundant organic substance on the earth with a simple chemical structure based on D-glucose linked through $\beta-1 \rightarrow 4$ linkage (Fig. 1). Therefore, it is a promising renewable resource for chemicals and fuels. However, in spite of such a simple chemical structure, effective conversion of cellulose into specific low molecular-weight substances for the utilization as chemicals or fuels is difficult. Pyrolysis is an effective method for converting cellulosic biomass into carbonized, gaseous and liquid substances ${ }^{6-8}$.

Cellulose pyrolysis was the subject of extensive studies over the past several decades. The previous studies on mechanism

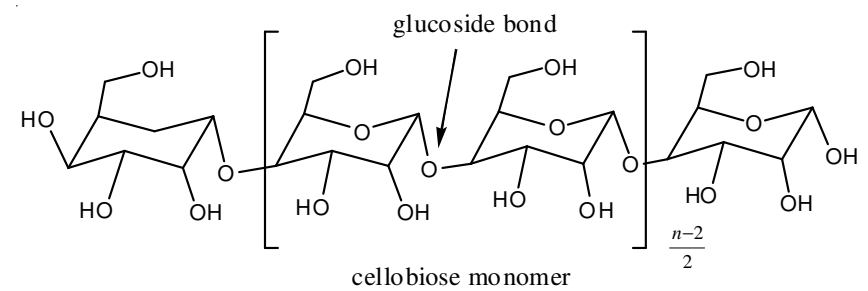

Fig. 1. Structural formula of cellulose chain

of cellulose pyrolysis mainly focused on experimental studies to explore the rate of tar, gases and coke in different conditions based on B-S model ${ }^{9-11}$. The thermogravimetric analyses were also performed to confirm pyrolytic characteristics of cellulose and the kinetic models of cellulose pyrolysis were constructed to obtain kinetic parameters ${ }^{12,13}$. However, there are few researches about chemical reactions, formation mechanisms of the main products and evolutionary processes of intermediates during cellulose pyrolysis ${ }^{14}$. In order to understand the mechanism of thermal decomposition of cellulose at the molecular level, the pyrolysis processes of the cellulose single molecule, of which polymerization degree is 10 , have been simulated by molecular dynamics method and the possible formation pathways of major products through reactions of all kinds of molecular fragments were analyzed in this study. 
SIMULATION CALCULATION METHOD

Before the thermal decomposition simulation, the molecular structure must be optimized. The process of looking for the global energy minimum on potential energy surface is called energy optimization and the optimized structure with the minimum potential energy is characteristic of the most stable conformation of molecular. In this paper the structure of single molecular cellulose with 10 monomers was optimized using a semi-empirical method AM1. First, the cellulose molecule was heated to a high temperature in order to make the structure of molecules stretch and relax fully at high temperature and then was cooled down to obtain optimal molecular structure.

After the cellulose molecule was optimized, we can get the original molecular size: $8.105(\AA) \times 6.255(\AA) \times 45.533(\AA)$ and simulation box size was set as $15(\AA) \times 15(\AA) \times 50(\AA)$. The pyrolysis processes were simulated in periodic boundary conditions by using AMBER force field. In the course of simulation the bond breakages were only considered and simulating temperature range was from initial temperature 273 to 1273 $\mathrm{K}$. The heating time was $100 \mathrm{ps}$ and simulating time was $10 \mathrm{ps}$ with time step of $0.001 \mathrm{ps}$. The value of energy (E) and temperature $(\mathrm{T})$ was extracted every 10 time steps. All simulation and computation were completed by using Hyperchem suite of programs.

\section{RESULTS AND DISCUSSION}

Optimized molecular structure: The optimized molecular structure is shown in Fig. 2. Partial optimized structure parameters of one circulation unit (namely cellobiose, Fig. 3) of cellulose are shown in Table-1. The Mulliken populations of bonds are shown in Table-2. Through the Mulliken populations of bonds we can understand the relative strength of the chemical bonds between atoms and know the most possible site of bonds cleavage. The smaller the Mulliken populations of bonds are, the easier to fracture the chemical bonds are. Table- 2 showed that the Mulliken populations of C-O bonds are smaller than that of $\mathrm{C}-\mathrm{C}$ bonds, so these bonds are easier to break than $\mathrm{C}-\mathrm{C}$ bonds.

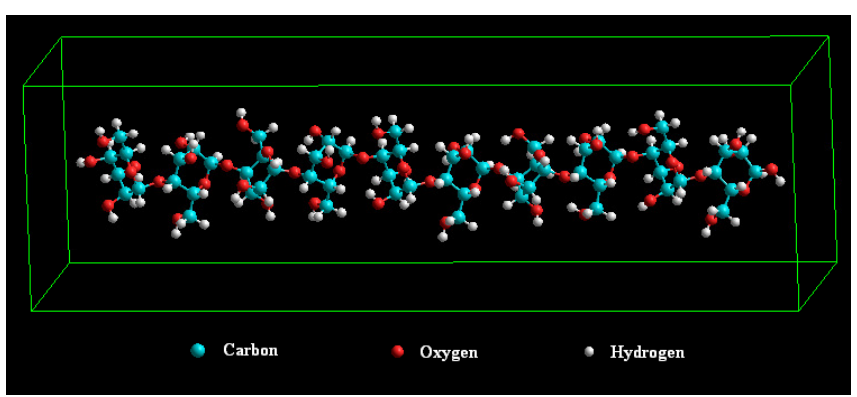

Fig. 2. Optimized structure for cellulose with 10 monomers<smiles>OCC1OC(O)C(O)C(O)C1OC1OC(CO)[C@@H](O)C(O)C1O</smiles>

Fig. 3. Cellulose circulation unit

\begin{tabular}{ccccccc}
\multicolumn{7}{c}{ TABLE-1 } \\
\multicolumn{7}{c}{ SOME OPTIMIZED STRUCTURE PARAMETERS } \\
OF A CIRCULATION UNIT OF CELLULOSE \\
\hline $\mathrm{R}(1,2)$ & $\mathrm{R}(2,3)$ & $\mathrm{R}(3,4)$ & $\mathrm{R}(4,5)$ & $\mathrm{R}(1,6)$ & $\mathrm{R}(1,7)$ & $\mathrm{R}(5,6)$ \\
1.538 & 1.546 & 1.545 & 1.540 & 1.443 & 1.532 & 1.409 \\
$\mathrm{R}(5,8)$ & $\mathrm{R}(2,18)$ & $\mathrm{R}(3,19)$ & $\mathrm{R}(4,20)$ & $\mathrm{R}(7,16)$ & $\mathrm{R}(8,11)$ & $\mathrm{R}(9,10)$ \\
1.432 & 1.422 & 1.405 & 1.422 & 1.422 & 1.429 & 1.538 \\
$\mathrm{R}(9,14)$ & $\mathrm{R}(10,11)$ & $\mathrm{R}(11,12)$ & $\mathrm{R}(12,15)$ & $\mathrm{R}(12,13)$ & $\mathrm{R}(13,14)$ & - \\
1.538 & 1.546 & 1.546 & 1.532 & 1.422 & 1.408 & - \\
\hline
\end{tabular}

Energy changes in the process of heating: Simulation system began to heat up from initial temperature $273 \mathrm{~K}$ and the temporal evolution of temperature and total energy (including kinetic energy and potential energy) is shown in Figs. 4 and 5. In the process of heating, the temperature and total energy of simulating cellulose molecule rise along with time.

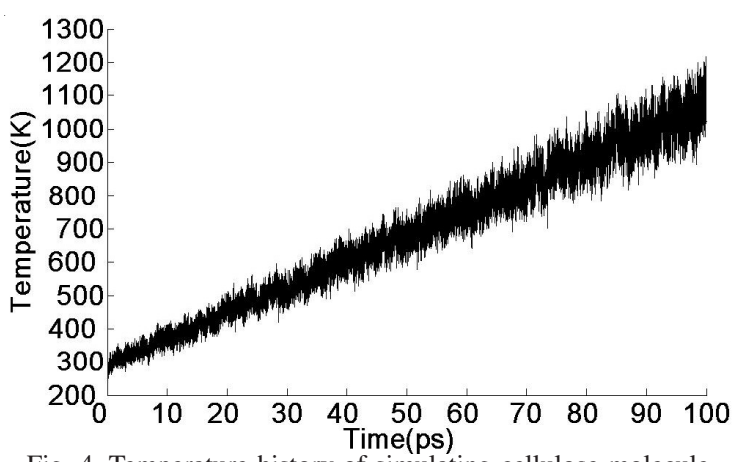

Fig. 4. Temperature history of simulating cellulose molecule

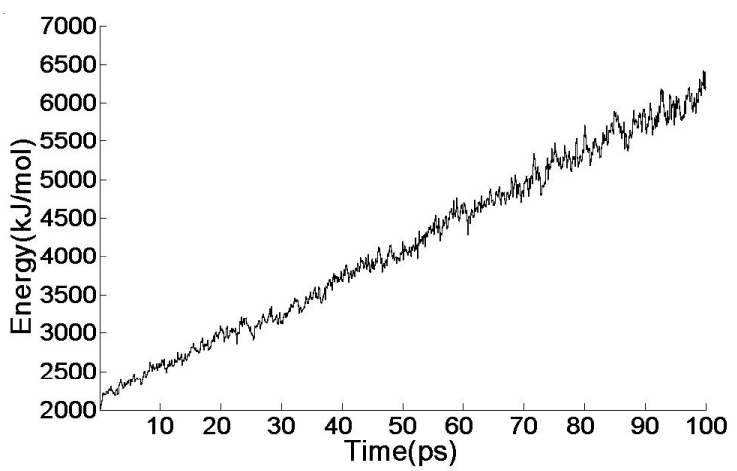

Fig. 5. Energy history of simulating cellulose molecule

\begin{tabular}{cccccc}
\multicolumn{7}{c}{ TABLE-2 } \\
MULLIKEN POPULATIONS OF BONDS OF A CIRCULATION UNIT OF CELLULOSE \\
\hline Bond & Mulliken population & Bond & Mulliken population & Bond & Mulliken population \\
\hline $\mathrm{C}(1)-\mathrm{C}(2)$ & 0.3146 & $\mathrm{C}(2)-\mathrm{O}(18)$ & 0.1628 & $\mathrm{C}(4)-\mathrm{C}(5)$ & 0.3471 \\
$\mathrm{C}(2)-\mathrm{C}(3)$ & 0.3298 & $\mathrm{C}(3)-\mathrm{O}(19)$ & 0.2033 & $\mathrm{C}(1)-\mathrm{C}(7)$ & 0.3307 \\
$\mathrm{C}(3)-\mathrm{C}(4)$ & 0.3168 & $\mathrm{C}(4)-\mathrm{O}(20)$ & 0.1896 & $\mathrm{C}(1)-\mathrm{O}(6)$ & 0.1852 \\
$\mathrm{C}(5)-\mathrm{O}(8)$ & 0.1304 & $\mathrm{C}(5)-\mathrm{O}(6)$ & 0.2170 & $\mathrm{C}(11)-\mathrm{O}(8)$ & 0.1655 \\
\hline
\end{tabular}


Breakage of molecular bonds: Thermogravimetric experiment analysis of cellulose showed that the thermal decomposition process of cellulose underwent three main stages $^{13}$. The first stage was low temperature pyrolysis process, in which there occurred tiny mass loss of cellulose and a small amount of dehydration happened. The second stage was intermediate temperature pyrolysis process, which is the main stage in the pyrolysis process. In intermediate temperature pyrolysis process, low molecular-weight gases and condensable volatiles were generated and there occurred obvious mass loss of cellulose. The last stage was high temperature pyrolysis process, in which the remnants underwent slow decomposition. The simulation process of pyrolysis of the cellulose single molecule can also be divided into three stages: low temperature stage, intermediate temperature stage and high temperature decomposition stage.

Low temperature decomposition stage (below $550 \mathrm{~K}$ ): During this stage the cellulose molecular structure occurs some physical changes and cellulose molecule absorbs heat energy in heating process, as a result, atomic bonds lengthen, bond angles and dihedral angles change and the molecular chain bends. There occur no molecular bonds breakage under 450 K. Above $450 \mathrm{~K}$, a small amount of hydroxyls begin to get out (Fig. 6).

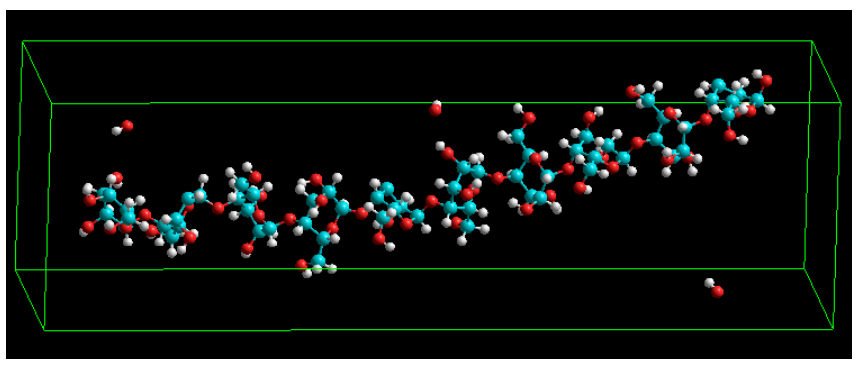

Fig. 6. Low temperature stage of cellulose pyrolysis

Intermediate temperature decomposition stage (550$800 \mathrm{~K})$ : A large number of bonds occur to break in this stage, which is the main stage in cellulose thermal decomposition. As the temperature increases, molecule group moves intensely and cellulose decomposes sharply (Fig. 7). The small molecular gases and condensable volatile matters are generated in the stage. The glycosidic bonds begin to break at $c a .600 \mathrm{~K}$, then the whole molecule depolymerizes and macromolecule fragments with different polymerization degree are formed. Macromolecule fragments further decompose to form cellulose monomers which will be converted to levoglucosan through reforming and dehydration. When the glycosidic bonds break, we can also find that internal bonds of cyclic monomer break to form all kinds of small molecular fragments at the same time. The open loop process of cyclic structure includes the breakage of C-C bond and hemiacetal C-O bond. The carbonyl and carboxyl functional groups can be generated through the breakage of hemiacetal C-O bond. The chemical compounds including carbonyl and carboxyl functional groups can decompose further to form $\mathrm{CO}$ and $\mathrm{CO}_{2}$. Large molecular fragments generated in thermal decomposition process are unstable and can be converted to various products through a series of reforming, dehydration, decarbonylation and other reactions.

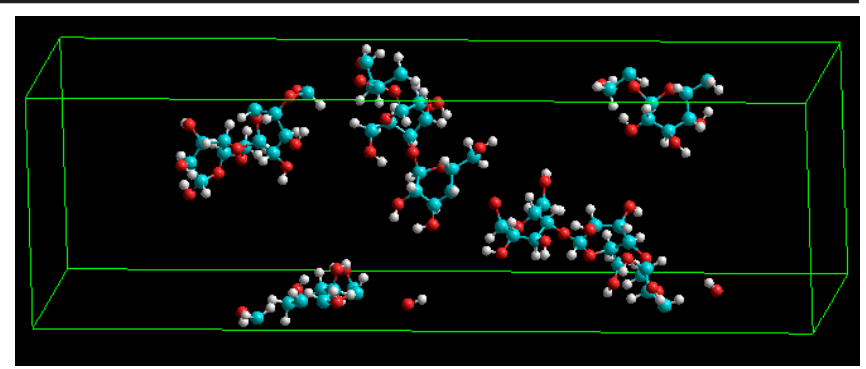

Fig. 7. Process of decomposition of cellulose at intermediate temperature

The experimental results showed ${ }^{10}$ that in intermediate temperature stage the main products are bio-oil, of which the main components are levoglucosan, glycolic-aldehyde, acetol etc. The above simulation results are consistent with the related experimental results ${ }^{9,10}$.

High temperature decomposition stage (above $800 \mathrm{~K}$ ): This stage is similar to the cellulose gasification process. During the simulation, due to the molecular fragments are not discharged and still remain in the simulation box, these molecular groups continue to decompose with the temperature increase, resulting in forming a large number of smaller molecular fragments (Fig. 8).

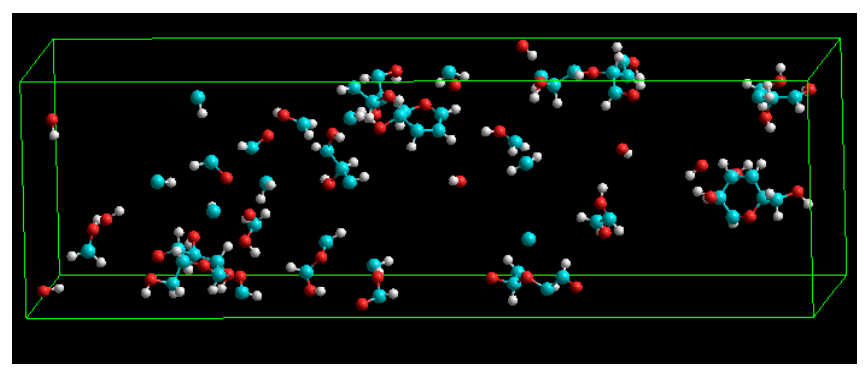

Fig. 8. Process of decomposition of cellulose at high temperature

From the above simulation results we can know that it is important to control thermal decomposition temperature to obtain different products. Biomass fast thermal decomposition experiment is mainly to get bio-oil, then the temperature must be controlled in intermediate temperature range and the products must be separated and cooled timely. Biomass gasification is mainly for gaseous products, so thermal decomposition temperature must be controlled in high temperature range to obtain more gaseous products.

Analysis of formation mechanism of major products: Cellulose fast thermal decomposition experimental results indicated that the main thermal decomposition products were bio-oil, gas and a little coke. The yield of highest bio-oil is up to $80 \%$ and the main components of bio-oil are organic compounds such as levoglucosan, glycolic-aldehyde, acetol, 5-hydroxymethyl furfural, furanose and toluene and the main components of gases are $\mathrm{CO}_{2}$ and $\mathrm{CO}^{9,10}$.

Richards ${ }^{15}$ considered that the heterolytic cleavage of glycosidic bond between glucose monomer lead to produce a considerable amount of levoglucosan $(1,6$-anhydro- $\beta$-Dglucopyranose) through transglycosylation reaction. In the simulation processes of pyrolysis, cellulose monomers are generated through glycosidic bonds breakage, which are further converted to levoglucosan through rearrangement and dehydration (Fig. 9). 


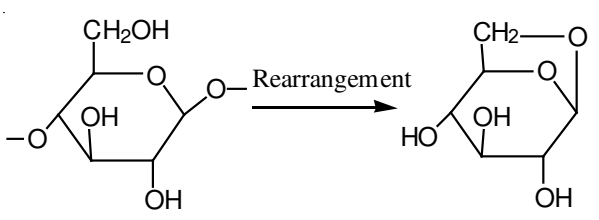

Fig. 9. Formation pathway of levoglucosan (LG)

Cellulose monomer can be also decomposed into a twocarbon molecule fragment and a four-carbon molecule fragment via ring-opening reaction. Two-carbon molecule fragment will reform and be converted to glycolic-aldehyde which is relatively stable (Fig. 10).

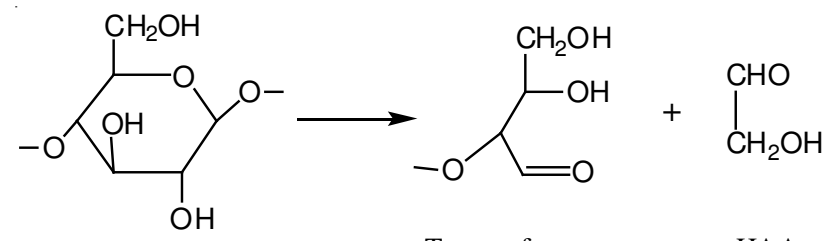

Cellulose monome

Tetrose fragment

HAA

Fig. 10. Formation pathway of glycolic-aldehyde (HAA) through pyranoid ring-opening

Four-carbon molecule fragment can be decomposed into various small molecule products through a series of reforming, dehydration, decarbonylation and other reactions. Because of the complexity of the chemical reaction, the decomposition of the four-carbon fragment has many different pathways. Fig. 11 gives a possible decomposition approach of the fourcarbon fragments.

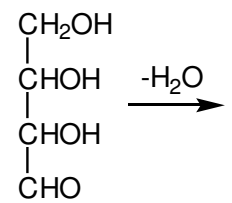<smiles>O=CC(O)CO</smiles><smiles>CC(C)(C)[C@H](O)C=O</smiles><smiles>CC(C)(CO)[O+]Cl</smiles>

Tetrose fragment

Fig. 11. Acetol

Six-carbon fragment are converted to furan ring structure via rearrangement and dehydration. As is shown in Fig. 12, six-carbon fragment undergoes three sequential dehydration reactions and generated the 5-hydroxymethyl furfural (5-HMF) which is an important product in cellulose decomposition.
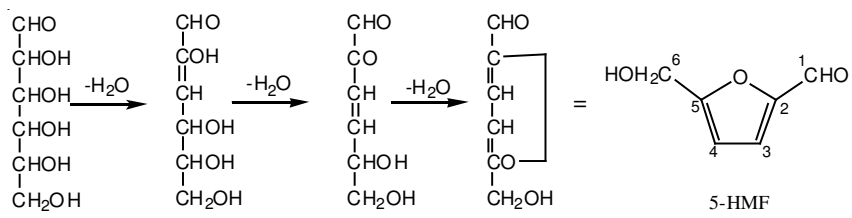

Fig. 12. Formation pathway of 5-HMF

The formation of furanose is after more change steps, which is similar to the formation of furfural. The glucopyranose is converted to acyclic carbonyl isomer via ring-opening reaction and acyclic carbonyl isomer is further converted to furanose structure through the bond of C(4)-C(1), then furanose undergoes the dehydration between hydroxys of C(1) and C(6) to produce 1,6-anhydro-furanose (Fig. 13).

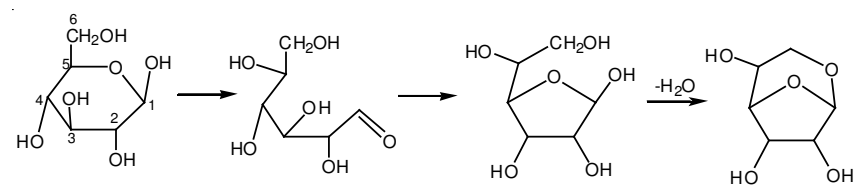

Fig. 13. Formation pathway of furanose

The formation of benzene ring structure can be obtained through condensation polymerization between aldehydes and ketones ${ }^{16}$. The benzene ring structure can be formed via polycondensation and cyclization between condensation compounds of acetaldehyde and acraldehyde (Fig. 14), the structure is further converted to phenol, toluene and other chemical compounds.

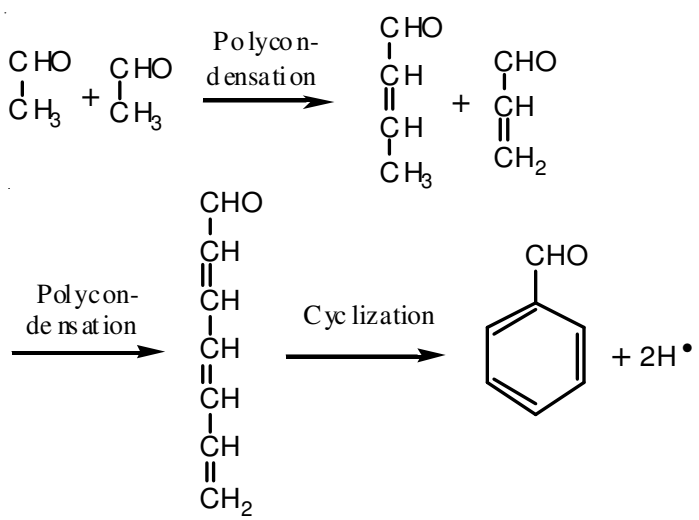

Fig. 14. Formation pathway of benzene ring through polycondensation

Because of the existence of aldehyde functional groups, many products with aldehyde functional groups are further decomposed to release $\mathrm{CO}$. Comparing with $\mathrm{CO}$, the yield of $\mathrm{CO}_{2}$ is low obviously. It is generally believed that the formation of carboxyl group rely on reforming of ketene structure in decomposition products ${ }^{17}$. For example, 2,3-hydroxyl-propionaldehyde is converted to ketene structure via dehydration and the ketene structure is extremely unstable and easily converted to carboxyl compound in the participation of water. Then carboxyl compound is further decomposed to release $\mathrm{CO}_{2}$ (Fig. 15).

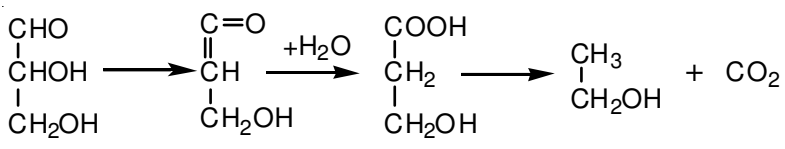

Fig. 15. Formation pathway of $\mathrm{CO}_{2}$

Because the components of cellulose thermal decomposition products are complex, above analysis only involve the possible formation pathways of some main products. We can see from thermal decomposition simulation results that the molecular fragments produced in thermal decomposition are very complex and various fragments can be converted to all kinds of products through various approaches, which lead to the complexity of thermal decomposition products.

\section{Conclusion}

The cellulose single molecular was simulated by molecular dynamic method in order to understand the reaction processes of cellulose thermal decomposition from the microscopic 
perspective. In the simulation processes, we find that hydroxyl bonds begin to break when temperature reaches $450 \mathrm{~K}$. With the increase of temperature, at $c a$. $600 \mathrm{~K}$ cellulose monomers get to be formed by glucoside bonds rupture and pyranoid rings are opened and all kinds of molecular fragments are generated at the same time. The thermal decomposition law obtained in simulation is basically consistent with the experimental results. Based on related experimental results in references, the possible formation pathways of major products through reactions of all kinds of molecular fragments were analyzed.

\section{ACKNOWLEDGEMENTS}

This work was supported by the National Natural Science Foundation of China (No. 51266002 ), the Science and Technology Funds of Guizhou province (No. [2012]2188), the Open Research Funds of State Key Laboratory of Pulp and Paper Engineering, South China University of Technology (No. 200928) and the Collaborative Funds of Guizhou Science and Technology Department and Guizhou University for Nationalities (No. LKM[2011]22).

\section{REFERENCES}

1. S.H. Shuit, K.T. Tan, K.T. Lee and A.H. Kamaruddin, Energy, 34, 1225 (2009).
2. J. Huang, C. Liu, X. Huang and H. Li, Comp. Theor. Chem., 964, 207 (2011).

3. E. Wetterlund, K. Pettersson and S. Harvey, Energy, 36, 932 (2011).

4. G.J. Kwon, D.Y. Kim, S. Kimura and S. Kuga, J. Anal. Appl. Pyrol., 80, 1 (2007).

5. R. Bassilakis, R.M. Carangelo and M.A. Wojtowicz, Fuel, 80, 1765 (2001).

6. Q. Lu, X. Zhu, Q. Li, Q. Guo and Q. Zhu, Prog. Chem., 19, 1064 (2007).

7. Z. Luo, S. Wang, Y. Liao, J. Zhou, Y. Gu and K. Cen, Biomass Bioenerg., 26, 455 (2004).

8. A.V. Bridgwater and G.V.C. Peacocke, Renew. Sust. Energ. Rev., 4, 1 (2000).

9. Y. Liao, Z. Luo, S. Wang, C. Yu and K. Cen, J. Fuel Chem. Technol., 31, 133 (2003).

10. Y. Liao, S. Wang, Z. Luo, H. Tan, C. Yu and J. Zhou, J. Zhejiang Univ. (Eng. Sci.), 5, 582 (2003).

11. J. Piskorz, D. Radlein and D.S. Scot, J. Anal. Appl. Pyrol., 9, 121 (1986).

12. T. Sonobe and N. Worasuwannarak, Fuel, 87, 414 (2008).

13. V. Mamleev, S. Bourbigot and J. Yvon, J. Anal. Appl. Pyrol., 80, 151 (2007).

14. J. Huang, C. Liu and S. Wei, Acta. Chim. Sin., 67, 2081 (2009).

15. G.N. Richards, J. Anal. Appl. Pyrol., 10, 251 (1987).

16. I.J. Miller and E.R. Saunders, Fuel, 66, 130 (1987).

17. J.L. Banyasz, S. Li, J. Lyons-Hart and K.H. Shafer, Fuel, 80, 1757 (2001). 